\title{
ANALYSIS OF EXPERIMENTAL AND NUMERICAL RESULTS OF A TURBULENT SWIRLING FLOW IN A TUBE
}

\author{
W.F.J. SAMPERS, A.P.G.G. LAMERS and A.A. van STEENHOVEN \\ Eindhoven University of Technology \\ Department of Mechanical Engineering \\ P.O. Box 513, 5600 MB Eindhoven, The Netherlands
}

(Received November 23 1992; in final form May 2 1993)

In many applications swirling flow is used to enhance heat and mass transfer. One of the problems of modelling a turbulent swirling flow is the choice of the turbulent closure model which is acceptable for engineering purposes. To evaluate which model performs best, numerical results are compared with experimental data. Local velocity measurements are carried out on a swirling flow in a circular tube. The measurement method is hot-wire anemometry combined with visualization techniques. The numerical analysis is carried out using the $k-\varepsilon$ model and the Algebraic Stress Model. In the experiment a symmetrical swirl was observed. Comparing the experimental data with the numerical results shows that the Algebraic Stress Model represents the experimental data quite well, whereas the $k-\varepsilon$ model fails.

KEYWORDS $k-\varepsilon$ moḍel Algebraic Stress Model Swirling flow Hot-wire anemometry.

\section{INTRODUCTION}

In combustion and other equipment, turbulent swirl is often used to enhance heat and mass transfer. This indicates the need for an accurate mathematical description of this type of flow. Many descriptions have been developed for turbulent flow phenomena e.g. Prandtl mixing length model, $k-\varepsilon$ model, Algebraic Stress Model (ASM), and Reynolds Stress Model (RSM). The first three models are generally available. Only the last three models are expected to give reasonable results. ${ }^{1,2,3}$

The aim of this study is to evaluate the standard $k-\varepsilon$ model and the ASM by means of experiments. The Prandtl mixing length model is not expected to give accurate results because a turbulent length-scale needs to be produced for the whole flow field. The RSM is considered too complex and expensive for practical applications. Similar experiments have been conducted by Kitoh. ${ }^{4}$ Kitoh did not compare his experiments with calculated results. His experiments concentrated on the decay of the swirl along a tube and on the local character of turbulence phenomena. We are mainly interested in the velocity distribution in a number of downstream cross sections of the tube and in the global character of turbulence, expressed in the turbulent kinetic energy. 


\section{EXPERIMENTAL SET-UP}

The flow in the test-rig (Figure 1) has a Reynolds number of 10,000 based on the bulk-velocity $u_{m}$ measured by means of an orifice. The airflow in the test-rig is $0.22 \mathrm{~m}^{3} / \mathrm{s}$ with an average bulk velocity $u_{m}$ of $7.5 \mathrm{~m} / \mathrm{s}$. The design of the swirl-generator is shown in Figure 2. The cross-sections where the velocity profiles are measured are located downstream from the swirl-generator at a distance of 9 , 10 and 18 pipe diameters respectively. At those positions a facility is made to insert a probe. In the probe a hot-wire sensor is mounted. At each cross-section 32 measuring points are placed on two orthogonal axes. The flow parameters of interest are the mean velocity components in axial, radial and tangential direction, the turbulent kinetic energy and the swirl number. The turbulent kinetic energy $k_{t}$ is directly calculated from the fluctuations of the velocity components written as $u_{z, r, \varphi}^{\prime}$

$$
k_{t}=\frac{1}{2}\left(u_{z}^{\prime 2}+u_{r}^{\prime 2}+u_{\varphi}^{\prime 2}\right)
$$

From the axial and tangential velocity distributions the swirl-number in a cross-section is calculated giving information on the intensity of the swirl. The

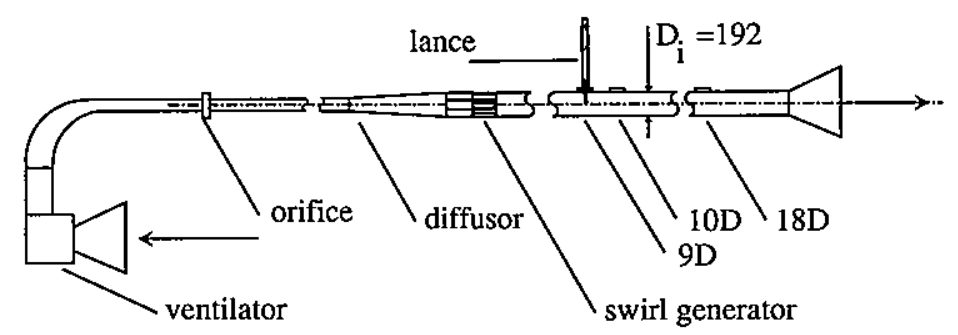

FIGURE 1 Experimental test-rig with ventilator, orifice, diffusor, swirl generator and test section with hot-wire lance. The inner diameter $D_{i}$ of the test section is given in $\mathrm{mm}$.

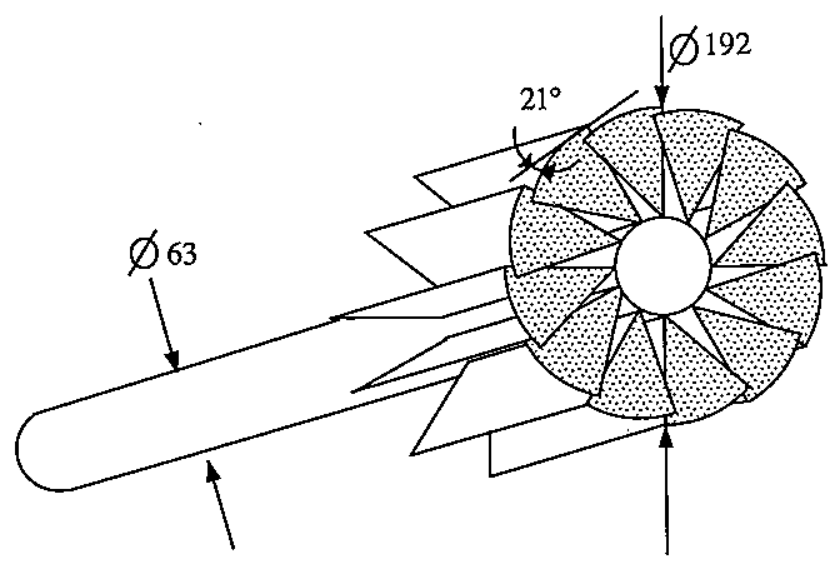

FIGURE 2 The swirl generator. Twelve equally spaced blades are bent to vanes under $21^{\circ}$ with a plane perpendicular to the axis. The dimensions are in $\mathrm{mm}$. 


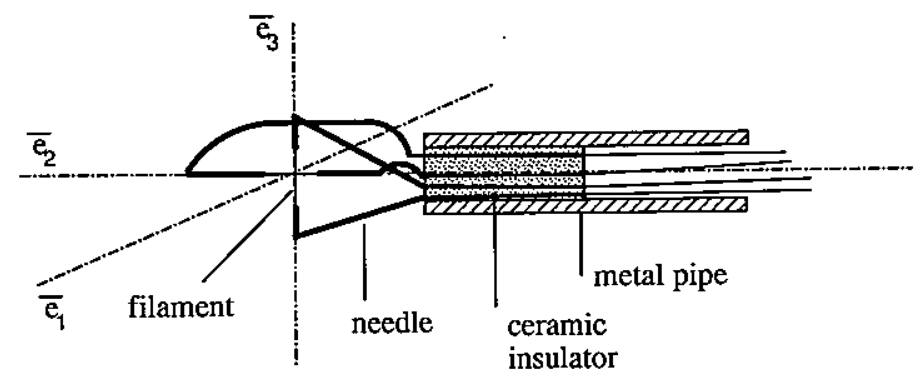

FIGURE 3 The cross-wire sensor. The metal pipe, ceramic insulator, support needles and platinized filaments are indicated. $\bar{e}_{1}, \bar{e}_{2}$ and $\bar{e}_{3}$ are the axes of a local coordinate system.

swirl-number $\Omega$ is defined as ${ }^{4}$

$$
\Omega=2 \pi \rho \int_{0}^{r_{0}} \frac{r^{2} u_{z} u_{\varphi}}{\pi \rho r_{0}^{3} u_{z}^{2}} d r
$$

Here are $u_{z, \varphi}$ the mean axial and tangential velocities. The radius of the tube $r_{0}$ is $96 \mathrm{~mm}$.

To measure the velocity components a hot-wire sensor, whose design is shown in Figure 3 , is used. It consists of two orthogonally placed platinized tungsten wires (diameter: $5 \mu \mathrm{m}$, selective length: $2 \mathrm{~mm}$, aspect ratio $1 / d: 400$ ). The signals from the anemometers are correlated to the instantaneous velocities through Jörgenson's relation, ${ }^{5}$ yielding for a single wire

$$
u_{\text {eff }}^{2}=u_{z}^{2}+k^{2} u_{r}^{2}+h^{2} u_{\varphi}^{2}
$$

where $u_{\text {eff }}$ is the effective velocity "felt" by the wire. The yawfactor $k$ and the pitchfactor $h$ are introduced to correct for cooling of the wire due to other velocity components than the component perpendicular to the wire (Figure 4).

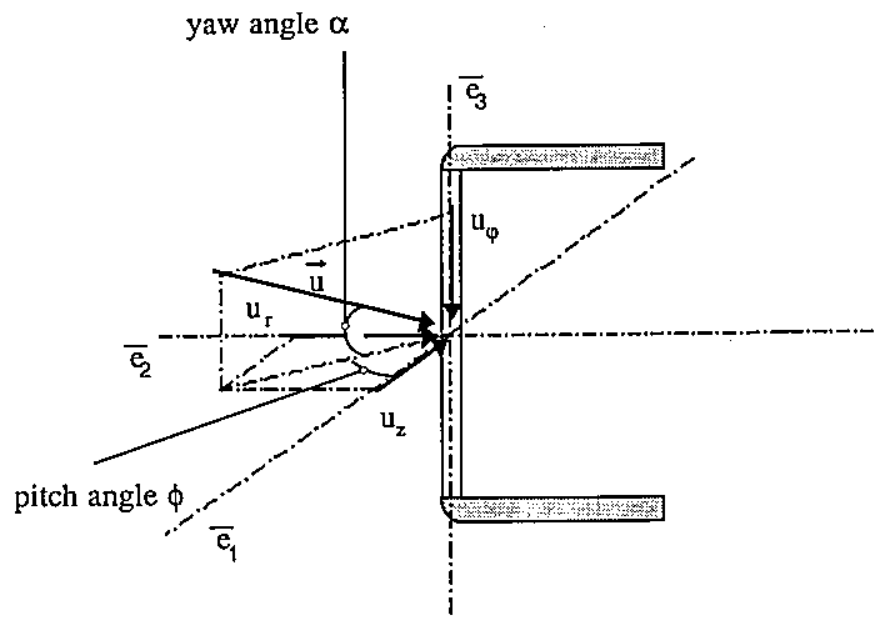

FIGURE 4 The yaw angle $\alpha$ and the pitch angle $\phi . \bar{e}_{1}, \bar{e}_{2}$ and $\bar{e}_{3}$ are the axes of a local coordinate system. 
the yaw and pitch factors are calibrated as function of the yaw angle $\alpha$ and the pitch angle $\phi$. Placing the wire succesively on one of three axes of a local orthogonal coordinate system the following matrix equation per measuring point is derived

$$
\left(\begin{array}{c}
u_{z}^{2} \\
u_{r}^{2} \\
u_{\varphi}^{2}
\end{array}\right)=\left(\begin{array}{ccc}
1 & h^{2} & k^{2} \\
1 & k^{2} & h^{2} \\
k^{2} & h^{2} & 1
\end{array}\right)^{-1} *\left(\begin{array}{c}
u_{\mathrm{eff}, \bar{e}_{1}}^{2} \\
u_{\mathrm{eff}, \bar{e}_{2}}^{2} \\
u_{\mathrm{eff}, \bar{e}_{3}}^{2}
\end{array}\right)
$$

The subscripts $\bar{e}_{1}, \bar{e}_{2}, \bar{e}_{3}$ denote the direction of the wire. By expression (4) the magnitude and the direction of the velocity vector are determined. The sign, however, is not defined due to the measuring principle of cooling. For each measuring point the sign is found by means of simple visualization techniques using smoke and tufts. The anemometer used is a DISA Type 55D00 Universal Anemometer, whose signals are converted by a Tecmar Labmaster A/D converter. The converted signals are modified and stored in a PC-AT using the software package ASYST. At each measurement point 100,000 samples were taken with a frequency of $20 \mathrm{kHz}$.

\section{NUMERICAL SIMULATION}

To solve complex physical problems like swirling flows a numerical approach is needed. Hence space must be divided into discrete elements setting limits to refinement. The high-frequency and spatially very small fluctuations of turbulent flow variables cannot be quantified directly. Consequently, each flow variable is split into a time mean component and a superimposed fluctuating component. If the terms in the basic equations are split in this way, mean values of the products of the velocity fluctuations appear, acting as additional shear stresses, the so-called Reynolds stresses. For these unknown turbulence correlations, assumptions must be made. Essentially, this entails the formulation of further conservation equations. A good compromise at the present time between acceptable computer processing times and accuracy of the simulation of turbulent flow phenomena is the $k-\varepsilon$ model. This model consists of two additional differential equations for the turbulent kinetic energy $k$ and the dissipation rate $\varepsilon$. However the $k-\varepsilon$ model considers the turbulence to be isotropic. In a turbulent swirling flow the turbulence phenomena are expected to be anisotropic. The Algebraic Stress Model is developed from the Reynolds Stress transport equations in order to provide a simpler model accounting for anisotropic effects.

Steady state turbulent flow in a tube can be described by conservation equations for time mean quantities. The time mean continuity and momentum equations can be found in Kuo. ${ }^{6}$ The derivation of the transport equations for turbulent kinetic energy $k$ and dissipation rate $\varepsilon$ is also given by Kuo. ${ }^{6}$ The kinematic turbulent viscosity, being a coupling between $k$ and $\varepsilon$, is given by

$$
v_{t}=\frac{C_{\mu} k^{2}}{\varepsilon}
$$


where the turbulent constant $C_{\mu}=0.09$. For the empirical constants in the $k$ and $\varepsilon$ equation the standard values are taken.

The ASM is developed from the RSM in order to provide a simpler model accounting for anisotropy. The terms containing gradients of the Reynolds stresses in the RSM are replaced by algebraic relations. ${ }^{7,8}$

Besides the three algebraic relations, the equations for the turbulent kinetic energy $k$ and the dissipation rate $\varepsilon$ need to be solved. A complete set of equations expressed in cylindrical coordinates can be found in Kim. ${ }^{3}$ For the constants used in the ASM equations the standard values are taken. The ASM is rather complex and some problems with the stability of the numerical solution exist but it accounts for anisotropy and is expected to be able to cope with more complex flows, like swirling flows, than the $k-\varepsilon$ model.

The models presented above are only valid for fully turbulent flows. Close to solid walls and at interfaces there are inevitable regions where the local Reynolds number is so small that the viscous effects predominate over turbulent ones. To be able to account for the viscous effects and for the large gradients of the variables near the walls, the so-called log-law function method of Launder and Spalding ${ }^{8,9}$ has been used. The variation of variables near the wall is taken to be similar to the variation found in two-dimensional boundary layers. The value of $k$ near the wall $k_{p}$, is supposed to be known from the regular balance equation for $k$. In calculating $k_{p}$, it is necessary to assign a value to the energy dissipation rate $\varepsilon$. Near the wall, an assumption is made for the value of $\varepsilon_{p}{ }^{8,9}$

The inlet boundary conditions for velocity and turbulent energy are derived from the measurements. The dissipation rate at the inlet is coupled to the turbulent energy according to

$$
\varepsilon_{\text {inlet }}=\frac{k_{\text {inlet }}^{1.5}}{0.5 r_{0}}
$$

At the outlet the normal gradients are set zero and the overal continuity balance is checked.

The numerical experiments are carried out in a 2-D axisymmetrical cylindrical domain with cyclic boundary conditions in tangential direction. The partial differential equations for the conservation of mass, momentum and the $k-\varepsilon$ resp. the ASM equations together with the boundary conditions and auxiliary relations form a closed set of equations. The governing equations can be expressed in a common form

$$
\nabla\left(u_{i} \phi\right)=\nabla\left(\Gamma_{\phi} \nabla \phi\right)+S_{\phi}
$$

where $\phi$ stands for the dependent variables, $\Gamma_{\phi}$ for the exchange coefficient of the variable $\phi$ and $S_{\phi}$ for the source term.

The numerical analysis is performed with both the $k-\varepsilon$ model and the ASM model. Equations of the form of Eq. (7) are solved by means of the finite volume package Fluent. The variables calculated are the mean velocity components in $z, r$ and $\varphi$ direction, the pressure field, the turbulent kinetic energy and the dissipation rate of turbulent energy and in case of ASM the other Reynold's stresses. 
The calculations are performed with a constant grid of 18 cells in the axial and 28 cells in the radial direction. First the solution is calculated with the $k-\varepsilon$ model taking about 700 iterations. This result is used as an initial flow field for the calculations with the ASM which needed 10,000-15,000 iterations on a micro VAX machine taking a calculation time of about one day.

\section{COMPARISON BETWEEN CALCULATIONS AND MEASUREMENTS}

The velocity and turbulent energy profiles are measured at 6 cross-sections in total but only the results at 9,10 and 18 pipe diameters from the swirl-generator are presented in this paper. The first measured position is taken at 9-D where we expect that most local disturbances due to the swirl generator are damped. The inlet profiles are derived from the experimental data of the first measurement position, at 9-D from the swirl generator. To compare the numerical data of the axisymmetrical 2-D problem definition, all measuring data of the two orthogonal lines $\left(0^{\circ}\right.$ and $\left.90^{\circ}\right)$ are averaged. The results of the measured inlet profiles as a function of the radius are presented in Figure 5. These results are used as inlet boundary conditions for the calculations.

In Figure 6, the measured and calculated axial velocity profiles with the $k-\varepsilon$ model and the ASM at 10 and 18-D from the swirl generator are given. At 10-D both models predict the measurements quite well. At 18-D the $k-\varepsilon$ model predicts an almost fully developed turbulent flow profile. The ASM approximates the measured profile more accurately. However at the wall and at the axis the differences are still large. The calculated radial profiles at 10-D and 18-D are almost zero for both models (Figure 7) whereas the measurements show a level between 1.5 and $2.5 \mathrm{~m} / \mathrm{s}$. The tangential velocity profiles are shown in Figure 8. The result calculated by the $k-\varepsilon$ model would indicate that the swirl is dissipated quickly; $25 \%$ of the initial value at $10-\mathrm{D}$ and $80 \%$ at $18-\mathrm{D}$. The measurements show that in reality the swirl is not dissipated so fast. The ASM approximates the

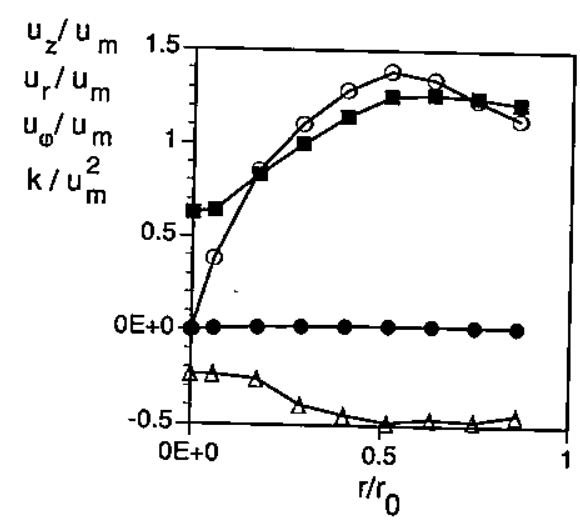

FIGURE 5 The measured inlet profiles of the test section as function of the radius at 9-D from the swirl generator. Normalised axial velocity $(\square)$, normalised radial velocity $(\Delta)$, normalised tangential
velocity $(O)$ and normalised turbulent kinetic energy $(\bullet)$. 


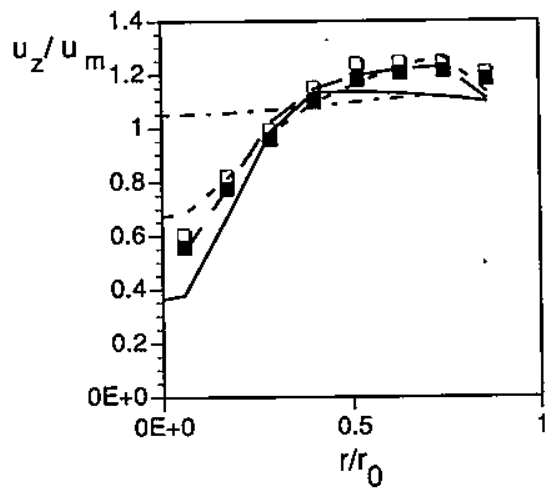

FIGURE 6 The measured and calculated axial velocity component as function of the radius. measured at $10-\mathrm{D},--k-\varepsilon$ at $10-\mathrm{D},---\mathrm{ASM}$ at $10-\mathrm{D}, \mathbf{m}$ measured at $18-\mathrm{D},--k-\varepsilon$ at $18-\mathrm{D}$, ASM at $18-\mathrm{D}$.

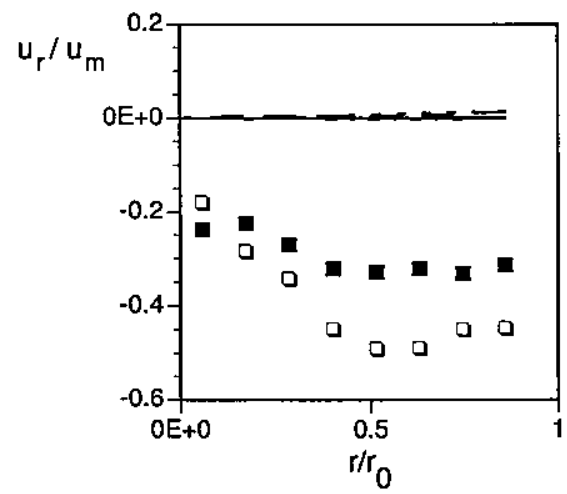

FIGURE 7 The measured and calculated radial velocity component as function of the radius. $\square$ measured at $10-\mathrm{D}, \cdots-k-\varepsilon$ at $10-\mathrm{D},-\ldots \mathrm{ASM}$ at $10-\mathrm{D}$, $\mathbf{m}$ measured at $18-\mathrm{D},-\cdots-\varepsilon$ at $18-\mathrm{D}$, ASM at $18-\mathrm{D}$.

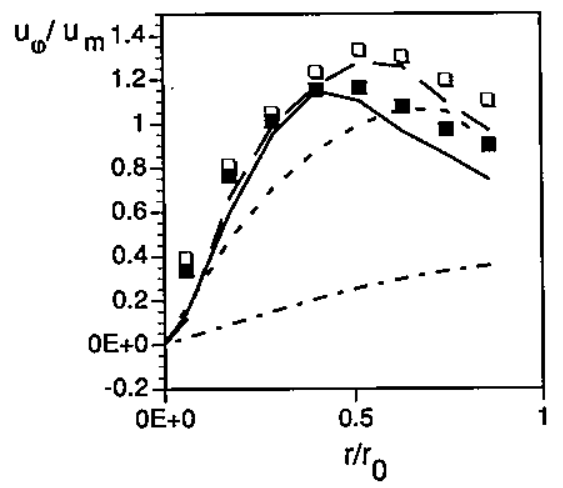

FIGURE 8 The measured and calculated tangential velocity component as function of the radius. $\square$

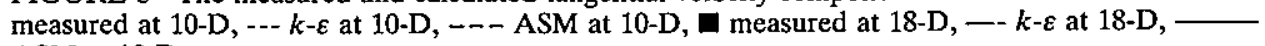
ASM at 18-D. 


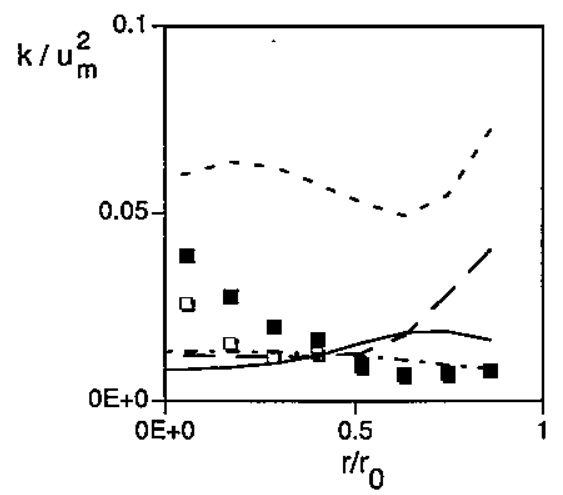

FIGURE 9 The measured and calculated turbulent kinetic energy as function of the radius. measured at $10-\mathrm{D},--k-\varepsilon$ at $10-\mathrm{D},---\mathrm{ASM}$ at $10-\mathrm{D}, \mathbf{\square}$ measured at $18-\mathrm{D},--k-\varepsilon$ at $18-\mathrm{D}$, ASM at 18-D.

measurement very well. At the wall however the difference with the measured profile is about $5 \%$ at $10-\mathrm{D}$ and $20 \%$ at $18-\mathrm{D}$. From this it is concluded that the modelling of the wall region is critical.

The detailed shape of the profiles of the turbulent energy shows large differences between measurement and calculation (Figure 9). In particular the $k-\varepsilon$ model gives large errors. In the calculations the turbulent energy is clearly dissipated close to the axis. Maybe due to instability of the vortex core this is a complex phenomena not modelled by 2-D formulation.

In Figure 10 the swirl numbers of the measurements and the calculations along the test-rig are compared. In case of the $k-\varepsilon$ model the swirl number is much lower than reality. The ASM predicts a comparable decay of the swirl number as is observed in the experiment.

The estimated relative errors of the measurements for $u, k$ and $\Omega$ are therefore respectively 12,24 and $23 \%$. These errors result from the addition of the error in the signal from the $A / D$ convertor $(3 \%)$ and the errors in the yaw and pitch factors $(5 \%)$.

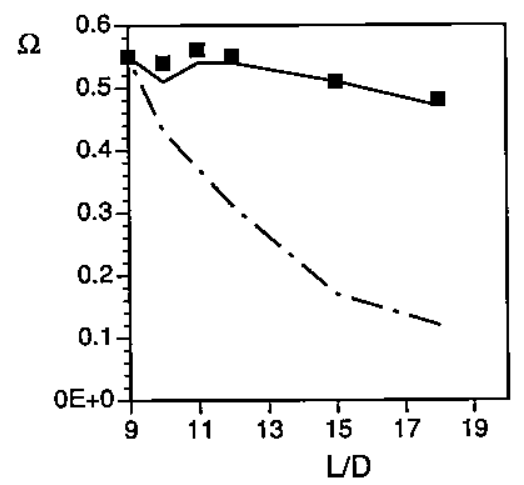

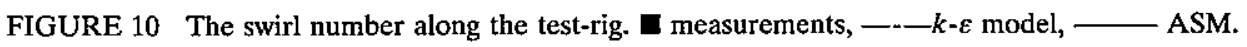




\section{CONCLUSIONS}

In three cross-sections of a measurement tube, at 9,10 resp. 18-D downstream of a swirl generator, the mean and fluctuating velocity components are measured with hot-wire anemometers. The measured profiles at 9-D were taken as inlet conditions for the numerical calculations with a $k-\varepsilon$ model and an ASM. The mean axial and tangential velocity components are calculated more accurately with ASM than with the $k-\varepsilon$ model. The discrepancy between the measured mean radial profile and the almost zero calculated profiles for both models is probably due to 3-D effects in the flow whereas the calculations are performed 2-D. On account of the measured radial velocity profile the superposition of a secondary flow on a fully developed flow pattern can be expected. The secondary flow leads to a pair of rotating swirls in the cross-section of the tube. Secondary flow patterns are found in experiments by Smithberg and Landis. ${ }^{10}$ The dissipation rate of turbulent kinetic energy shows significant differences for both models whereas the decay of the swirl-number is predicted correctly with ASM. In general it is clear that the ASM approximates the measured data best.

\section{NOMENCLATURE}

$C_{\mu} \quad$ constant in the $k-\varepsilon$ model

$D \quad$ diameter of the tube

$h \quad$ yaw factor

$k \quad$ pitch factor

$k \quad$ turbulent kinetic energy

$r \quad$ radial distance

$r_{0} \quad$ radius of the tube

Re Reynolds number $=\rho u_{m} D / \mu$

$S_{\phi} \quad$ source term of $\phi$-equation

$u_{\text {eff }} \quad$ effective velocity

$u_{m} \quad$ bulk velocity

$u_{z}, u_{r}, u_{\varphi} \quad$ instantaneous velocity component in axial, radial and tangential directions

\section{Greek Symbols}

$\begin{array}{ll}\varepsilon & \text { dissipation rate of turbulent energy } \\ \Omega & \text { swirl number } \\ \rho & \text { density } \\ v_{t} & \text { kinematic turbulent viscosity } \\ \mu & \text { dynamic viscosity }\end{array}$


$\phi \quad$ general variable

$\Gamma_{\phi} \quad$ exchange coefficient of the variable $\phi$

$\begin{array}{ll}\text { Indices } & \\ 0 & \text { reference value } \\ \text { eff } & \text { effective variable } \\ \text { inlet } & \text { fluctuating velocity component } \\ t & \text { index for the entrance } \\ p & \text { turbulent variable } \\ & \text { index for a value close to the wall }\end{array}$

\section{REFERENCES}

1. Khalil, E.E., and Assaf, H.M.W., Proc. Num. Meth. in Lam. and Turb. Flow, 363, Pineridge, Swansea (1981).

2. Boysan, F., and Swithenbank, J., Proc. Num. Meth. in Lam. and Turb. Flow, 425, Pineridge, Swansea (1981).

3. Kim, K.Y., and Chung, M.K., Int. J. Heat and Fluid Flow, 9, 62 (1988).

4. Kitoh, O., J. Fluid Mech., 225, 445 (1991).

5. Jorgenson, F.E., DISA info, 11, 31 (1971).

6. Kuo, K., Principles of combustion, Wiley, New York (1986).

7. Launder, B.E., Reece, G.H., and Rodi, W., J. Fluid Mech., 68, 537 (1975).

8. Launder, B.E., and Spalding, D.B., Comp. Meth. in Appl. Mech. and Eng., 3, 269 (1974).

9. Launder, B.E., and Spalding, D.B., Mathematical Models of Turbulence, Academic, London (1972).

10. Smithberg, E., and Landis, F., J. of Heat Transfer, Trans. of the ASME, 2, 39 (1964). 